\title{
Osteofitos de la primera articulación costocondral: respuesta protectora articular en deportistas de alto rendimiento
}

\author{
Osteophytes of the first costochondral joint: joint protective \\ response in high performance athletes
}

\author{
Luis Gerardo Domínguez Carrillo, ${ }^{*}$ Luis Gerardo Domínguez Gasca ${ }^{\ddagger}$ \\ Citar como: Domínguez CLG, Domínguez GLG. Osteofitos de la primera articulación costocondral: respuesta protectora \\ articular en deportistas de alto rendimiento. Acta Med. 2021; 19 (1): 130-131. https://dx.doi.org/10.35366/98584
}

Paciente masculino de 56 años, profesionista, durante su juventud futbolista en la posición de guardameta durante 18 años con carácter profesional. Se le efectuó estudio tele de tórax para valoración preoperatoria por motivos de cirugía

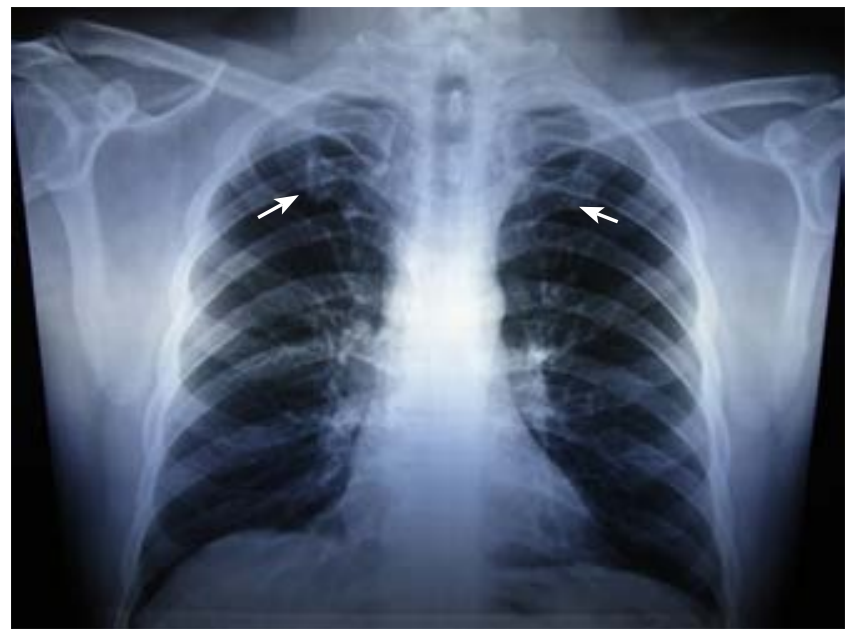

Figura 1: Radiografía tele de tórax donde como hallazgo se observa calcificación del cartílago de la primera articulación condrocostal y presencia de osteofitos (flechas) que indican existencia de pseudoartrosis bilateral asintomática en masculino de 56 años con antecedente de haber sido guardameta profesional. a nivel lumbar. Asintomático en cuanto a tórax (Figura 1 y 2). Llamó la atención presencia de osteofitos importantes en primera articulación condrocostal de manera bilateral.

La observación en humanos y modelos animales de que la formación de osteofitos puede ocurrir sin daño manifiesto del cartílago, parece indicar que su presencia y la osteoartritis no están necesariamente relacionadas entre sí, ya que la formación de osteofitos puede ocurrir en articulaciones "sanas" como resultado de estímulos mecánicos o envejecimiento.

La pseudoartrosis de la primera costilla es un hallazgo común en pacientes con calcificación del cartílago costal, es un hallazgo incidental frecuente y rara vez se ha informado que sea sintomática, es más común en hombres (40.6\%) que en mujeres (27.8\%), lo que se explica por los diferentes niveles de actividad de género. ${ }^{2}$

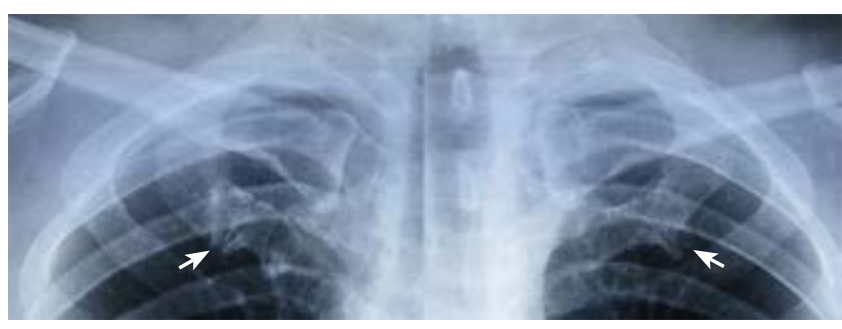

Figura 2: Acercamiento de la Figura 1.
* Especialista en Medicina de Rehabilitación. Catedrático de la Facultad de Medicina de León, Universidad de Guanajuato. México.

₹ Ortopedista. División de Cirugía del Hospital Ángeles León, León, Guanajuato. México.

\section{Correspondencia:}

Acad. Dr. Luis Gerardo Domínguez Carrillo

Correo electrónico: Igdominguez@hotmail.com

Aceptado: 06-02-2020.

www.medigraphic.com/actamedica 
En cuanto a la fisiopatogenia, conforme avanza la edad hay calcificación progresiva de estas partes cartilaginosas, lo que modifica la articulación pasando de sincondrosis a una sinostosis completa, pues las partes cartilaginosas de la primera costilla y su sincondrosis están involucradas en el funcionamiento adecuado del movimiento de la cintura escapular; si existe calcificación severa, la flexibilidad de las partes cartilaginosas disminuye, lo que puede conducir a la formación de pseudoartrosis para obtener el rango de movimiento. Otro factor que puede estar involucrado son las fracturas por estrés que se asocian con morbilidad mínima en la primera costilla, están relacionadas con el movimiento vigoroso repetitivo de la extremidad superior como se observa en los jugadores de béisbol, la práctica de remo y en otros atletas ${ }^{3}$ (como en el caso motivo de esta comunicación). Los efectos del trauma repetitivo en el hueso a menudo se describen en términos de daño por fatiga inducido por microtrauma, microgrietas y procesos de reparación ósea. La primera anomalía del cartílago costal observada parece representar una combinación de calcificación del cartílago costal relacionada con la edad y la respuesta del hueso al trauma crónico repetitivo asociado con el uso vigoroso y/o elevado de la extremidad superior, con presencia de osteofitos ${ }^{4}$ sin efectos negativos o incluso pueden tener efectos positivos (como en el caso presentado) al aumentar la superficie de la articulación.

En general, los osteofitos se desarrollan en las articulaciones diartrodiales; se piensa que los estímulos mecánicos que se transcriben a factores bioquímicos a nivel celular o estímulos bioquímicos autónomos inician el proceso de condrogénesis en el periostio y la membrana sinovial, donde las células similares a los macrófagos contribuyen en la formación de los osteofitos proporcionando factores de crecimiento. Los factores más importantes que inducen osteofitos son TGF $\beta$ y los factores BMP2. Los osteofitos inducidos por BMP2 se localizan en sitios de la articulación donde las placas de crecimiento se encuentran con el espacio articular, mientras que los osteofitos inducidos por TGF $\beta$ se originan en el periostio en sitios remotos de las placas de crecimiento. La localización y el patrón de desarrollo de los osteofitos inducidos por TGF $\beta$ son más similares a los osteofitos relacionados con la OA que los osteofitos inducidos por BMP. ${ }^{1}$

Radiológicamente, los criterios para pseudoartrosis ${ }^{5}$ son: a) estructuras similares a articulaciones con márgenes escleróticos; b) un espacio articular visible o hendiduras completas en las partes cartilaginosas de la primera costilla lateral a la articulación esternocostal y medial de la interfaz hueso-cartílago de la primera costilla y; c) la apariencia típica de las superficies articuladas "tipo osteofito". No obstante, dado el pequeño número de informes de casos de pseudoartrosis sintomática su relevancia clínica es limitada, pero debe formar parte del diagnóstico diferencial y no confundirse con patología tumoral en esta articulación.

\section{REFERENCIAS}

1. Van der Kraan MP, Van den Berg WB. Osteophytes: relevance and biology. Osteoarthritis Cartilage. 2007; 15: 237-244. doi: 10.1016/j. joca.2006.11.006.

2. Gossner J. Pseudarthrosis of the cartilaginous part of the first rib is a common incidental finding on chest CT. Diagn Interv Imaging. 2016; 97 (1): 53-56. doi: 10.1016/j. diii.2014.11.029.

3. Tarazona VP, Romo RR. Pseudarthrosis of the first rib in a patient who practices yoga. A case report. Acta Orthop Mex. 2009; 23 (6): 383-385.

4. Sheng GG, Duwayri YM, Emery VB, Wittenberg AM, Moriarty $\mathrm{CT}$, Thompson RW. Costochondral calcification, osteophytic degeneration, and occult first rib fractures in patients with venous thoracic outlet syndrome. J Vasc Surg. 2012; 55 (5): 1363-1369.

5. Schils JP, Resnick D, Haghighi P, Trudell D, Sartoris DJ. Sternocostal joints. Anatomic, radiographic and pathologic features in adult cadavers. Invest Radiol. 1989; 24 (8): 596-603. 